\title{
Anomalous dependence of response time on the electric field in an electroclinic liquid crystal with large induced tilt and polarization
}

\author{
U. Manna, ${ }^{1}$ Jang-Kun Song, ${ }^{1}$ J. K. Vij, ${ }^{1, a)}$ and J. Naciri ${ }^{2}$ \\ ${ }^{1}$ Department of Electronic and Electrical Engineering, Trinity College, University of Dublin, \\ Dublin 2, Ireland \\ ${ }^{2}$ Center for Bio/Molecular Science and Engineering, Naval Research Laboratory, \\ Washington, DC 20375, USA
}

(Received 25 September 2008; accepted 20 November 2008; published online 5 January 2009)

\begin{abstract}
We investigate the response time of an electroclinic liquid crystal with large induced tilt and polarization (de Vries smectic $A^{*}$ ). The response time is found to increase with the increase in electric field up to a threshold value in contrast with the general case of a decrease with field. This behavior is unusual and contrasts with that observed for a conventional smectic- $A^{*}$ phase. The anomalous behavior is explained by the Langevin process of the director reorientation by assuming an increase in the effective dipole moment $\left(\mu_{\text {eff }}\right)$ with field. The response time decreases with the increase in temperature in the smectic- $A^{*}$ phase. We find the existence of a finite correlation length of a few tens of nm which in both temperature and field dependent. (C) 2009 American Institute of Physics. [DOI: 10.1063/1.3049319]
\end{abstract}

An electric field $E$ applied parallel to the layer of a chiral smectic- $A^{*}\left(\operatorname{Sm} A^{*}\right)$ phase induces a molecular tilt $\theta$ relative to the layer normal in a plane orthogonal to the field, which is called the electroclinic effect. ${ }^{1}$ This can be described by a phenomenological model that predicts a linear dependence of the induced tilt angle $\theta$ on the applied field $E$ for low field strengths. This is given by

$$
\theta=c E / \alpha_{0}\left(T-T_{A C}\right),
$$

where $c$ is the electroclinic coupling constant, $\alpha_{0}$ is a phenomenological constant, and $T_{A C}$ is $\mathrm{Sm} A^{*}-\mathrm{Sm} C^{*}$ transition temperature. The electroclinic effect is a unique and technologically useful electro-optical property of a chiral $\mathrm{Sm} A *$ liquid crystal. Electroclinic liquid crystals have a potential for development of projection displays, spatial light modulators, optical correlators, smart pixel image processors, etc., where speed, high contrast, and gray scale are of the utmost importance. ${ }^{2-4}$ However, application of electric field in the $\mathrm{Sm} A^{*}$ phase parallel to the smectic layers induces stripes arising from a reduction in the layer spacing due to the molecular tilting and a consequent smectic layer buckling.,6 This has a detrimental effect on the contrast ratio of electrooptic devices. ${ }^{7,8}$ As a result, the $\operatorname{Sm} A^{*}$ materials having zero or low layer shrinkage with decreasing temperature or increasing field have drawn significant scientific interest due to their potential for applications in display and photonic devices. ${ }^{9}$ In the de Vries' "diffuse cone model" of these materials, the molecular long axis, and presumably the local director, is tilted by a finite angle. However, it is azimuthally randomized with uniform distribution $f(\varphi)=1 / 2 \pi$ on a cone from layer to layer as well as within a single layer, ${ }^{10-12}$ while the macroscopic director lies along the layer normal. Application of electric field tends to align the transverse dipoles, and this gives rise to a large electroclinic effect, the induced tilt, and the polarization. ${ }^{13-15}$

In this letter, we report an anomalous behavior of the response time in an electroclinic liquid crystal having small

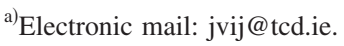

layer contraction, large induced tilt, and polarization. We find that the response time increases in the de Vries-type $\mathrm{Sm} A^{*}$ phase up to a certain value of the applied field. This behavior contrasts with that observed for a conventional $\operatorname{Sm} A^{*}$ phase. Upon assuming that the reorientation of the molecular dipoles with electric field in the de Vries-type $\operatorname{Sm} A^{*}$ phase is of the Langevin type, and by considering increase in the effective molecular dipole moment $\left(\mu_{\text {eff }}\right)$ with increasing field in the Langevin equation, we show that the molecular correlation length $\left(\xi_{\perp}\right)$ increases with increasing field; consequently the response time in the de Vries-type $\operatorname{Sm} A^{*}$ phase increases with field.

The liquid crystalline compound 4-[3'nitro-4' -((R)-1-methylhexyloxy)phenyl]phenyl 4-(6heptylmethyltrisiloxyhexyloxy)Benzoate (TSiKN65), $\mathrm{SmC}^{*}$ $27{ }^{\circ} \mathrm{C} \mathrm{Sm} A^{*} 57^{\circ} \mathrm{C}$ I used for our study is reported as a prototype de Vries material having a small layer contraction with decreasing temperature and increasing electric field, large electroclinic effect, and small orientational order parameter. $^{13,16,17}$ Results of the optical birefringence, tilt angle, and the polarization measurements upon cooling a $9 \mu \mathrm{m}$ cell from the isotropic phase with and without the external electric field in TSiKN65 are shown in Fig. 1. If the helical structure is suppressed by the surface anchoring, which is usually the case in planar cells [confirmed by the optical texture of $\mathrm{SmC}^{*}$ at $26{ }^{\circ} \mathrm{C}$ in Fig. 1(b) since there are no disclination lines due to the helical structure], the $S$ order parameter may show a sudden increase because the randomly distributed liquid crystalline molecules in de Vries $\mathrm{Sm} A^{*}$ phase align along the same direction as in the $\mathrm{Sm}^{*}$ phase. As a result, the birefringence increases significantly upon transition from de Vries-type $\mathrm{Sm} A^{*}$ phase to $\mathrm{Sm} C^{*}$ phase. ${ }^{15,18}$ For TSiKN65, we find that the birefringence goes up by around $43 \%$ upon transition from $\mathrm{Sm} A^{*}$ to $\mathrm{SmC}^{*}$ phase, as shown in Fig. 1(d). By decreasing the temperature in the $\mathrm{SmC}^{*}$ phase, the birefringence decreases slowly due to the appearance of helical structure as shown by the disclination lines perpendicular to the rubbing direction in $\mathrm{SmC}^{*}$ at $23{ }^{\circ} \mathrm{C}$ in Fig. 1(c). The birefringence with applied field 
(a) $\mathrm{Sm}-A^{*} 30^{\circ} \mathrm{C}$
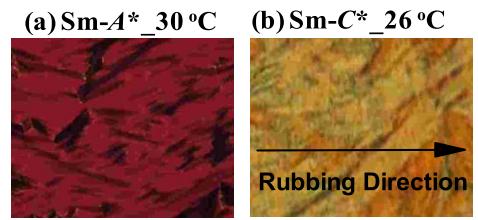

(c) $\mathrm{Sm}-\mathrm{C}^{*} 23^{\circ} \mathrm{C}$
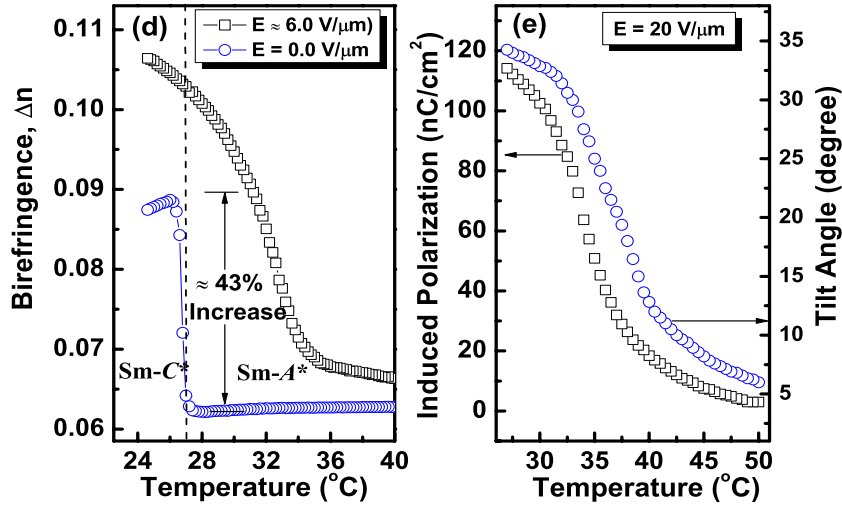

FIG. 1. (Color online) Optical textures of (a) $\mathrm{Sm} A^{*}$ phase at a temperature of $30{ }^{\circ} \mathrm{C}$, (b) $\mathrm{SmC} C^{*}$ phase at $26^{\circ} \mathrm{C}$, and (c) $\mathrm{SmC} C^{*}$ phase at $23{ }^{\circ} \mathrm{C}$. In the $\mathrm{SmC}^{*}$ phase at $23{ }^{\circ} \mathrm{C}$, disclination lines perpendicular to the rubbing direction due to helical structure are observed, whereas at $26{ }^{\circ} \mathrm{C}$ no such lines in the $\mathrm{SmC}^{*}$ phase are observed. (d) Temperature dependence of the optical birefringence with and without the electric field in the $\mathrm{Sm} A^{*}$ and $\mathrm{SmC}$ phases of TSiKN65. The birefringence without field is 0.062 at $28^{\circ} \mathrm{C}$ compared to 0.07 and with field of $5 \mathrm{~V} / \mu \mathrm{m}$ is 0.01 compared to 0.095 as in Ref. 17 (a small difference could be caused by a difference in the alignment). (e) Temperature dependence of the electric field induced polarization and the tilt angle of TSiKN65.

shows a continuous growth in the entire range of temperatures corresponding to the $\mathrm{Sm} A^{*}$ and $\mathrm{Sm} C^{*}$ phases. In the de Vries-type $\operatorname{Sm} A^{*}$ phase, the induced polarization and the tilt angle are due to the biasing of the molecular azimuthal distribution perpendicular to the electric field in addition to the usual electroclinic effect. As a result, the polarization and the tilt angle continue to grow with the electric field in the $\mathrm{Sm} A^{*}$ phase of TSiKN65, as shown in Fig. 1(e). The field induced polarization and the apparent tilt angle in the $\mathrm{Sm} A^{*}$ phase are as large as $112 \mathrm{nC} \mathrm{cm}^{-2}$ and $34^{\circ}$ at a temperature of $27.5^{\circ} \mathrm{C}$, respectively.

For response time measurements, the aligned cell was placed on a hot stage that is controlled to a temperature with an accuracy better than $\pm 0.01 \mathrm{~K}$. In this configuration, the transmitted light intensity through the cell varies as

$$
I=I_{0} \sin ^{2}\left[2\left(\theta+\theta_{0}\right)\right],
$$

where $\theta$ is the field induced tilt angle and $\theta_{0}$ is the angle between the liquid crystalline optic axis and the polarizer at zero field. After determining the dark state, $\theta_{0}$ was set to $22.5^{\circ}$ for optimum sensitivity. We measured the response time (rise time) of the transmitted optical power (10\% $\rightarrow 90 \%$ ) detected with a photodiode whose output was amplified and monitored on an oscilloscope subject to a dc balanced $10 \mathrm{~Hz}$ square wave. The characteristic response time in the $\mathrm{Sm} A^{*}$ phase is given by

$$
\tau=\eta_{\theta} / \alpha_{0}\left(T-T_{C}\right),
$$

where $\eta_{\theta}$ is the rotational viscosity associated with the $\theta$ motion. Figure 2 presents the dependence of the response time on electric field in the $\operatorname{Sm} A^{*}$ phase for TSiKN65 at various temperatures. We find that the response time increases, which continues to be the case to a certain value of

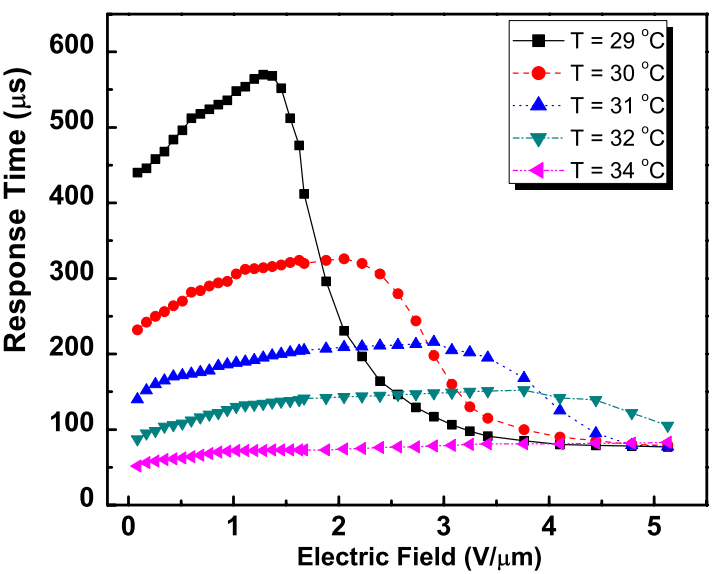

FIG. 2. (Color online) Electric field dependent response time in the $\operatorname{Sm} A^{*}$ phase of TSiKN65 for various temperatures.

the electric field $\left(E_{c}\right)$ for all temperatures. Upon further increasing the electric field, the response time decreases. This behavior contrasts with that for a conventional $\mathrm{Sm} A^{*}$ phase, where the response time is independent of $E$ in the small $\theta$ approximation and has a small dependence on electric field for larger $\theta,^{3,19}$ as observed in KN125., ${ }^{40}$ For higher temperatures within $\mathrm{Sm} A^{*}$ phase in TSiKN65, it was found that the response time increases to a certain value of the electric field, ${ }^{3,4}$ but the reasons were not given nor was it highlighted as an anomalous property of the material. The observed unusual behavior of the field dependent response time can well be explained by the de Vries scenario upon assuming that the reorientation of the molecular dipoles with the electric field is of the Langevin type and by considering an increase in the effective dipole moment $\left(\mu_{\text {eff }}\right)$ with increasing field as discussed below.

With the application of $E$, the apparent optic axis rotates from 0 (with $\langle\cos \varphi\rangle=0,\left\langle\cos ^{2} \varphi\right\rangle=1 / 2$ ) to $\theta_{A}$ (with $\langle\cos \varphi\rangle$ $\left.\approx 1,\left\langle\cos ^{2} \varphi\right\rangle \approx 1\right)$, where $\theta_{A}$ is the smectic cone angle. The averages $\langle\cos \varphi\rangle$ and $\left\langle\cos ^{2} \varphi\right\rangle$ can be evaluated using the Langevin equation for the azimuthal probability distribution described by the ratio of aligning energy $\mu_{\mathrm{eff}} E$ by electric field to the thermal energy $k_{B} T$, and is given by the

$$
P_{s}=P_{l}\langle\cos \varphi\rangle=\frac{\int_{o}^{\pi} \exp \left(\frac{\mu_{\mathrm{eff}} E \cos \varphi}{k_{B} T}\right)\left(P_{l} \cos \varphi\right) d \varphi}{\int_{o}^{\pi} \exp \left(\frac{\mu_{\mathrm{eff}} E \cos \varphi}{k_{B} T}\right) d \varphi},
$$

where $k_{B}$ is the Boltzmann constant, $P_{s}\left(\approx P_{l}\right.$, local polarization at high field) is the field induced macroscopic polarization, and $\mu_{\text {eff }}$ is the effective dipole moment given by

$$
\mu_{\mathrm{eff}}=\pi d\left(\xi_{\perp} / 2\right)^{2} P_{l},
$$

where $\xi_{\perp}$ is the molecular correlation length in a single layer and $d$ is smectic layer thickness. Note that the Langevin process described above assumes $\mu_{\text {eff }}$ as constant for different values of $E$. Figure 3(a) shows the electric field dependent $P_{s}$ for different temperatures. The black solid lines in Fig. 3(a) are the best fits of the Langevin theory, described by Eq. (4), to the experimental results. The observed results are simulated for values of $\mu_{\mathrm{eff}}$ varying from approximately 4 to 2000 


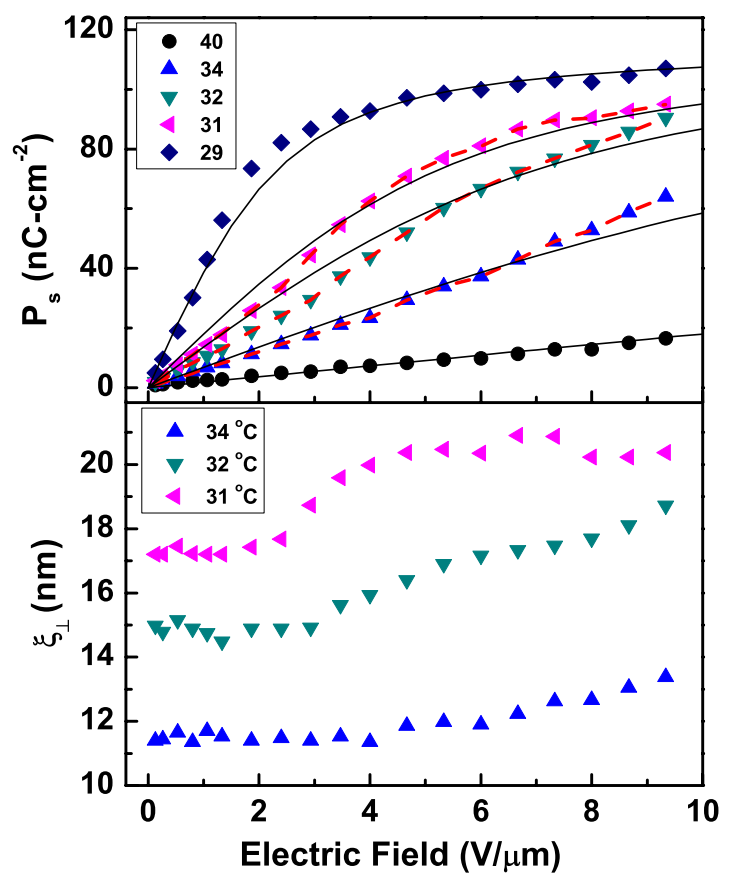

FIG. 3. (Color online) (a) represents the electric field induced polarization $\left(P_{s}\right)$ for different temperatures in TSiKN65. The solid black lines are the best fits of the data to the Langevin equation described in Eq. (4). (b) represents the electric field dependent molecular correlation length in a single layer in the de Vries-type $\mathrm{Sm} A^{*}$ phase determined by fitting the electric field dependent $P_{s}$, as shown by red dashed lines in (a), to the Langevin process by considering the increase in $\mu_{\text {eff }}$ with the field.

D depending on the temperature in the de Vries-type $\operatorname{Sm} A^{*}$ phase. The temperature dependent values of $\mu_{\text {eff }}$ correspond to a finite value of $\xi_{\perp}$, calculated from Eq. (5) and shown in Fig. 3(b) at zero electric field for 34,32 , and $31^{\circ} \mathrm{C}$. Hence, we can conclude that the molecules in the de Vries-type $\mathrm{Sm} A^{*}$ phase collectively respond to an electric field with a finite value of correlation length rather than individually as described in de Vries' model. For lower temperatures, the value of correlation length is higher. As a result, the response time increases especially for lower temperatures. The Langevin process described by Eq. (4) does not consider an increase in the $\mu_{\text {eff }}$ with electric field, which may not be the case for de Vries $\mathrm{Sm} A^{*}$ since application of the field aligns the transverse dipoles of these molecules. This is confirmed by achieving a better fitting of the field dependent $P_{s}$ to the Langevin equation for temperatures of 34,32 , and $31^{\circ} \mathrm{C}$ as shown by red dashed lines in Fig. 3(a), where the increase in $\mu_{\text {eff }}$ with field is considered. From the field dependent $\mu_{\text {eff }}$ values for which the $P_{s}$ are simulated, we find that upon increasing the field $\xi_{\perp}$ increases, as shown in Fig. 3(b); as a result, the response time increases with field. The same phenomenon is responsible for the increase in the dielectric strength with field in the $\mathrm{Sm} A^{*}$ phase of TSiKN65. ${ }^{21}$

To summarize, we find that there exists a finite correlation length even in the absence of electric field in a de Vriestype $\operatorname{Sm} A^{*}$ phase, where the molecules are tilted with the same angle and direction. By increasing the electric field applied across the cell, the correlation length increases. As a result, the response time increases in the de Vries $\operatorname{Sm} A^{*}$ phase initially with an increase in the field. The correlation length is also found to increase with a decrease in temperature in the $\operatorname{Sm} A^{*}$ phase.

The work was partially funded by SFI (Grant Nos. 02/ IN.1/I.031 and RFP06/RFP/ENE039).

${ }^{1}$ S. Garoff and R. B. Meyer, Phys. Rev. A 19, 338 (1979).

${ }^{2}$ K. Eguchi, U.S. Patent No. 5,514,426 (7 May 1996).

${ }^{3}$ J. Naciri, J. Ruth, G. Crawford, R. Shashidhar, and B. R. Ratna, Chem. Mater. 7, 1397 (1995).

${ }^{4}$ B. R. Ratna, K. S. Nelson, H. Li, C. Wilson, and J. Naciri, Proc. SPIE 3297, 2 (1998).

${ }^{5}$ J. Pavel and M. Glogarova, Liq. Cryst. 9, 87 (1991).

${ }^{6}$ G. P. Crawford, R. E. Geer, J. Naciri, R. Shashidhar, and B. R. Ratna, Appl. Phys. Lett. 65, 2937 (1994).

${ }^{7}$ R. Shashidhar, J. Naciri, and B. R. Ratna, Adv. Chem. Phys. 113, 51 (2000).

${ }^{8}$ J. R. Lindle, F. J. Bartoli, S. R. Flom, A. T. Harter, B. R. Ratna, and R. Shashidhar, Appl. Phys. Lett. 70, 1536 (1997).

${ }^{9}$ D. M. Walba, E. Korblova, L. Eshdat, M. C. Biewer, H. Yang, C. Jones, M. Nakata, M. Talarico, R. Shao, and N. A. Clark, J. Soc. Inf. Disp. 15, 585 (2007).

${ }^{10}$ A. de Vries, Mol. Cryst. Liq. Cryst. 41, 27 (1977).

${ }^{11}$ A. de Vries, J. Chem. Phys. 71, 25 (1979).

${ }^{12}$ J. P. F. Lagerwall and F. Giesselmann, ChemPhysChem 7, 20 (2006)

${ }^{13}$ M. S. Spector, P. A. Heiney, J. Naciri, B. T. Weslowski, D. B. Holt, and R. Shashidhar, Phys. Rev. E 61, 1579 (2000).

${ }^{14}$ N. A. Clark, T. Bellini, R.-F. Shao, D. Coleman, S. Bardon, D. R. Link, J E. Maclennan, X.-H. Chen, M. D. Wand, D. M. Walba, P. Rudquist, and S. T. Lagerwall, Appl. Phys. Lett. 80, 4097 (2002).

${ }^{15}$ J. P. F. Lagerwall, F. Giesselmann, and M. D. Radcliffe, Phys. Rev. E 66, 031703 (2002).

${ }^{16}$ N. Hayashi, A. Kocot, M. J. Linehan, A. Fukuda, J. K. Vij, G. Heppke, J. Naciri, S. Kawada, and S. Kondoh, Phys. Rev. E 74, 051706 (2006).

${ }^{17}$ J. V. Selinger, P. J. Collings, and R. Shashidhar, Phys. Rev. E 64, 061705 (2001).

${ }^{18}$ U. Manna, J. K. Song, Yu. P. Panarin, A. Fukuda, and J. K. Vij, Phys. Rev. E 77, 041707 (2008).

${ }^{19}$ I. Abdulhalim and G. Moddel, Liq. Cryst. 9, 493 (1991).

${ }^{20}$ A. Hermanns, C. M. Wilson, J. Y. Patel, J. W. Naciri, J. R. Lindle, and B. R. Ratna, Appl. Phys. Lett. 73, 3644 (1998).

${ }^{21}$ U. Manna, J. K. Song, J. K. Vij, and J. Naciri, Phys. Rev. E 78, 041705 (2008). 